\title{
Atmospheric Depth Models in the Field of View of LHAASO-WFCTA
}

\section{J. J. $\mathbf{X i a}^{a, *}$ and F. R. Zhu ${ }^{a}$}

${ }^{a}$ School of Physical Science and Technology, Southwest Jiaotong University, 611756, Chengdu, China

E-mail: zhufr@home.swjtu.edu.cn

\begin{abstract}
:
The Wide Field-of-view Cherenkov Telescope Array (WFCTA) is one of the main components of the Large High Altitude Air Shower Observatory(LHAASO) located in Haizi Mountain,Daocheng County, Sichuan Province. WFCTA probes cosmic rays by observing Cherenkov photons generated by secondary particles in Extensive Air showers.Changes in atmosphere depth will lead to changes in the detection efficiency of WFCTA. Therefore, the variation of atmosphere depth plays an essential role in the calibration of WFCTA. In this proceeding,the temporal variation of atmosphere depth at LHAASO site is studied with the MSIS-90 atmosphere model,and compared with the American standard atmosphere model,Saber satellite experimental data, and observational data collected at the LHAASO site. MSIS-90 is used to obtain monthly atmosphere depth at the LHAASO site.
\end{abstract}

$37^{\text {th }}$ International Cosmic Ray Conference (ICRC 2021)

July 12 th - 23rd, 2021

Online - Berlin, Germany

${ }^{*}$ Presenter 


\section{Introduction}

LHAASO is a dual-task facility designed for Cosmic-Ray (CR) and Gamma-Ray studies at $\mathrm{TeV}$ and $\mathrm{PeV}$ energies. It consists of three interconnected detectors, Water Cherenkov Detector Array (WCDA), Kilometer Square Array (KM2A), and Wide Field of-view Cherenkov Telescope Array (WFCTA), located at $4410 \mathrm{~m}$ above sea level in the Sichuan province of China .WFCTA is mainly used to observe the Cherenkov or fluorescence light generated by secondary particles produced by cosmic rays entering the atmosphere to probe the primary cosmic rays[1].

Atmospheric depth refers to the mass of the atmosphere per unit area, which is equal to the mass of the atmosphere in the vertical air column extending upward to the upper boundary of the atmosphere per unit area, and the unit is $\mathrm{g} / \mathrm{cm}^{2}$. The thickness of the atmosphere at one standard atmosphere at sea level is $10306 \mathrm{~g} / \mathrm{cm}^{2}$.

In order to simplify the calculation, J. Linsley proposed the global American Standard Atmosphere Model.The density variation of the atmosphere with altitude is modeled by 5 layers. In the lower four of them the density follows an exponential dependence on the altitude leading to a relation between the mass overburden $\mathrm{T}(\mathrm{h})$ of the atmosphere and the height $\mathrm{h}$ of the form[2]

$$
\mathrm{T}(h)=a_{i}+b_{i} \exp \left(-\frac{h_{i}}{c_{i}}\right)
$$

In the fifth layer the mass overburden decreases linearly with height

$$
\mathrm{T}(h)=a_{i}-b_{i} \cdot \frac{h_{i}}{c_{i}}
$$

This model is often used in the estimation of high-energy cosmic rays shower. However, the Cherenkov radiation or fluorescence in the WFCTA field of view will change with the change of atmosphere depth over time. Therefore, it is very important to quantitatively study the change of atmosphere depth over time in the LHAASO-WFCTA field of view to reduce the systematic error of LHAASO-WFCTA and improve the data accuracy. At present, other experiments observing Cherenkov light or fluorescence in the world mostly monitor the atmosphere depth. For example, on different dates in 1993, measurements over Stuttgart, transmitted by Deutscher Wetterdienst Offenbach, established seven typical Antarctic atmospheres

The Auger Observatory measured the depth of the atmosphere over a 12-month period to build a model of the depth for the atmosphere at their site. The MSIS-90 model describes the neutral temperature and density in the Earth's atmosphere from the ground to the height of the thermosphere. Below $72.5 \mathrm{~km}$, the model is mainly based on the regional averaged temperature and pressure tabulated in the MAP manual drawn by Barnett and Corney. Below 20 kilometers, these data are supplemented by averages from the National Meteorological Center (NMC). Above 72.5 kilometers, the model takes into account the data obtained from the space shuttle flight and the updated incoherent scattering results[3].Compared with the US standard atmosphere model, it can describe the temporal and spatial changes of atmosphere density and temperature[4].In this proceeding, the LHAASO-MSIS model is established by using MSIS-90 model data. 


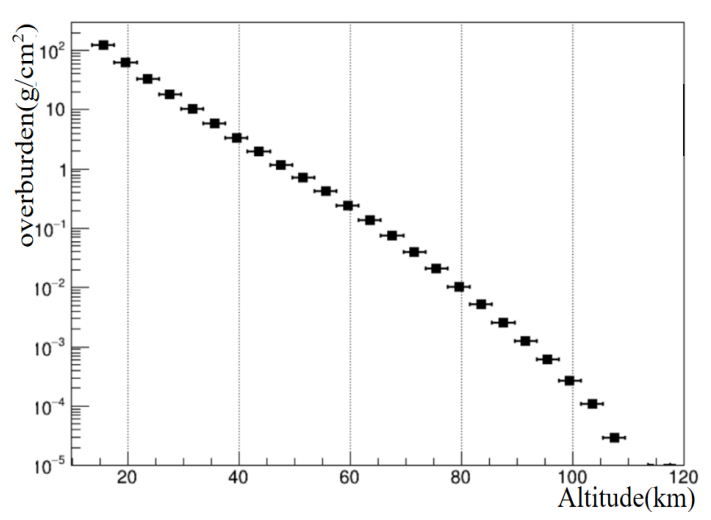

(a)

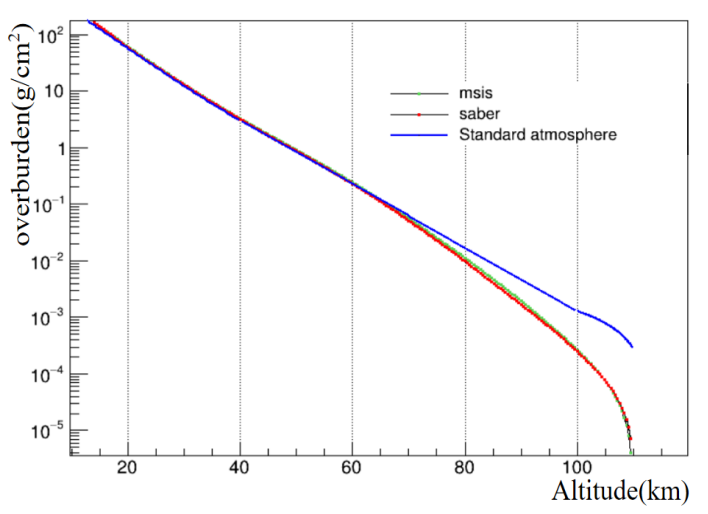

(b)

Figure 1: (a)The average of the 31-day SABER data; (b)Comparison of Standard atmosphere model, the SABER data and the MSIS model, where the MSIS model has the same times as the SABER data.

\section{Atmospheric depth of experimental data}

The TIMED (Thermosphere Ionosphere Mesosphere Energetics and Dynamics) satellite was launched by NASA in July 2001. Its main instrument include a multi-channel infrared radiometer (Sounding of the Atmosphere Using Broadband Emission Radiometry, SABER). The orbit period of the TIMED satellite is $1.6 \mathrm{~h}$. It runs about 15 orbits every day and survey the coverage observation of the earth once every 60 days.The SABER also measures the temperature in the altitude range of about $14-140 \mathrm{~km}$, the vertical resolution is about $2 \mathrm{~km}$ and the horizontal resolution is about $400 \mathrm{~km}[5]$. The mesosphere density measured by MARMOT lidar in Golmud by the Institute of atmosphere Physics, Chinese Academy of Sciences is in good agreement with the measured data from TIMED/SABER satellites and the model value in the middle atmosphere is $1 \%$ larger than the measured values from lidar and satellite data $20 \%[6]$.

In this proceeding, we downloaded a total of 31 days SABER data from 2013 to 2018. The atmosphere density is integrated in the vertical direction to calculate the atmosphere depth at LHAASO. After averaging the obtained data, and plotting the standard error of the average, the result is shown in Fig.1(a). It can be seen that the data ranges from 14 $\mathrm{km}$ to $110 \mathrm{~km}$ since the satellite scanning starts at $14 \mathrm{~km}$.

Fig.2(a) shows the difference between the satellite's atmosphere depth measurement and the MSIS model from 2013 to 2018. It can be seen that there is a large difference between 14 and $20 \mathrm{~km}$, and the maximum difference can reach $20 \mathrm{~g} / \mathrm{cm}^{2}$. This may be because the atmosphere depth of $14-20 \mathrm{~km}$ itself is relatively large. Fig.2(b) shows the ratio of atmosphere depth obtained the satellite data from 2013 to 2018. It can be seen that most of these ratios are within $20 \%$.

In order to better study the atmosphere changes at LHAASO, we use the atmosphere pressure data recorded by the standard weather station at LHAASO. The data is obtained every 5 minutes from January 2020 to August 2020(Fig.3(a)). The pressure data is divided 


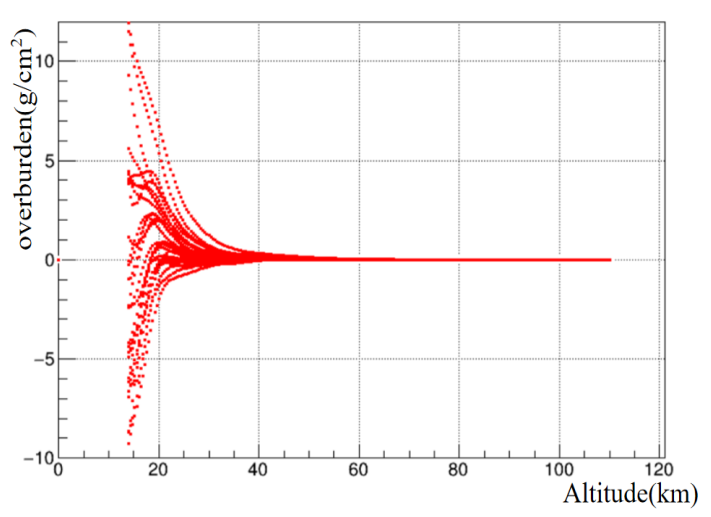

(a)

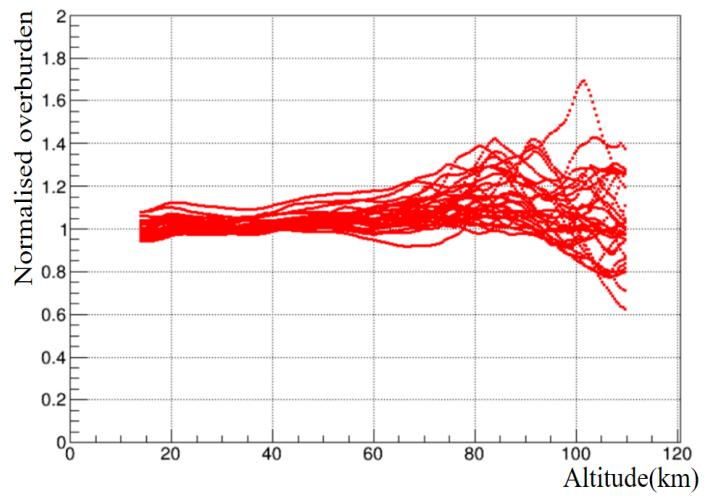

(b)

Figure 2: Comparison of atmosphere depth derived from SABER data and MSISE model in 31 days.(a) Variation of the difference with altitude;(b) the ratio of these depths.

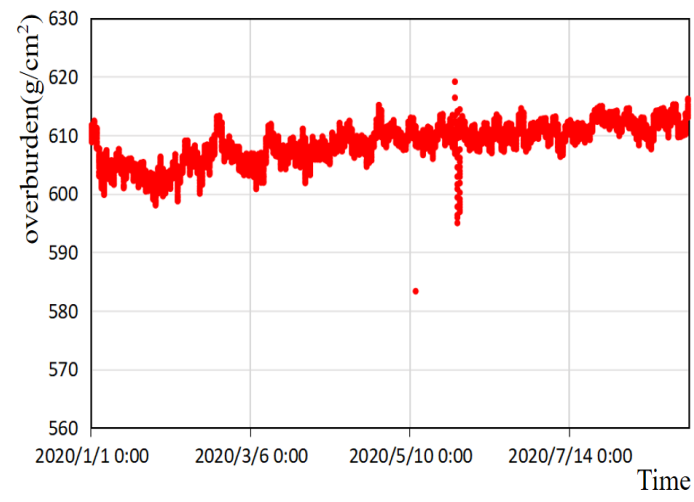

(a)

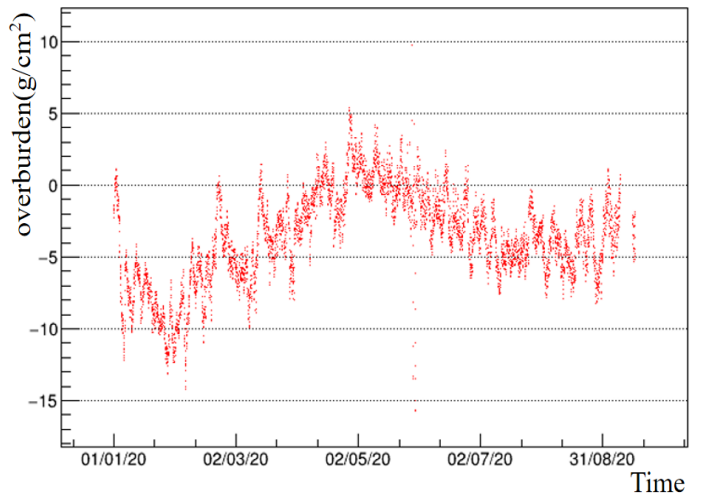

(b)

Figure 3: (a)Variation of surface atmospheric depth in Daocheng over time; (b)difference of atmospheric depth (standard weather station measurement-MSIS.)

by the corresponding acceleration of gravity to get the overburden.

At $4.4 \mathrm{~km}$, the measured data from January to August of 2020 are selected for comparison with the model data of the same period. Fig3(b) shows the difference between MSIS and measured data in the same period. The difference between the model value and the measured value is in the range of $0-15 \mathrm{~g} / \mathrm{cm} 2$. corresponding to a relative change of $1 \%-3 \%$, and the model value is greater than the measured value during most times.

\section{Establishment of LHAASO-Models}

In this proceeding, we first selected the MSIS-90 model density data from $4.4 \mathrm{~km}$ to $100 \mathrm{~km}$ in LHAASO site in 2018 and integrated the atmosphere density in the vertical direction to calculate the atmosphere depth. Fig.4(a)shows daily atmosphere depth in April 2018 normalized to that of April 1. It can be seen that the atmosphere depth is 


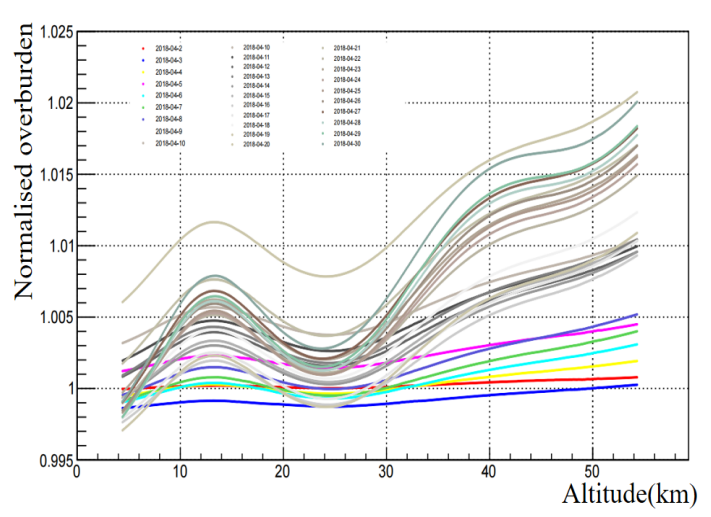

(a)

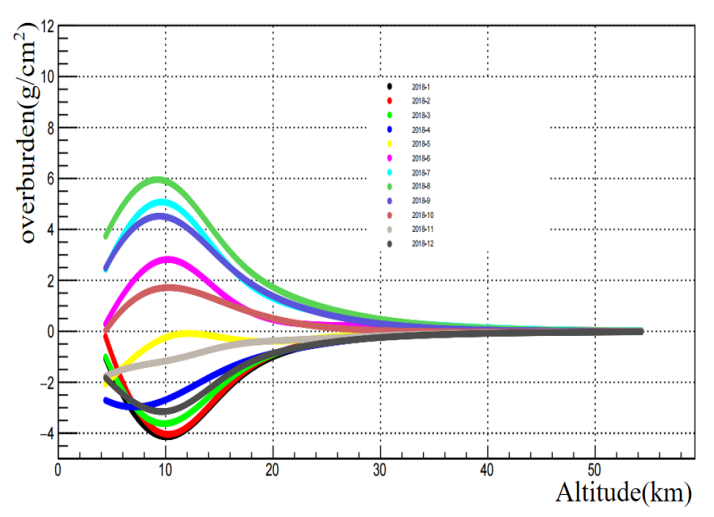

(b)

Figure 4: (a)Daily variation of atmosphere depth(April other day/April 1); (b) Monthly variation of atmosphere depth(different monthly average-annual average.)

changing gradually, and the maximum difference within a month is about $1 \%$. Comparing the monthly average data of atmosphere depth in 2018 with the annual average data, the monthly variation of atmosphere depth at LHAASO is shown in Fig.4(b). The observation time of WFCTA is at night. In order to improve the accuracy of fitting, we select MSIS data from 8:00 pm to 6:00 a.m. every month, and establish LHAASO models by using the fitting formula of American standard atmospheric models. The fitting results are shown in Table1.

\section{Results and Discussion}

In this proceeding, the LHAASO-MSIS model are established and compared the model with the American standard atmospheric model. It is found that the atmospheric depth values of the MSIS model from $4 \mathrm{~km}$ to $100 \mathrm{~km}$ at the LHAASO are all greater than the American standard atmosphere model. It was found that the MSIS model value was greater than the satellite measurement data at $14 \mathrm{~km}$ to $110 \mathrm{~km}$, and the difference was up to 20 $\mathrm{g} / \mathrm{cm}^{2}$. Then, the model data were compared with the meteorological station measurement data, and it was found that at $4.4 \mathrm{~km}$, the model data were larger than the experimental data, the absolute error fluctuation range was 0-15 g/cm2, and the relative error range was 1\%-2\%. Based on the above data, we fitted the MSIS data in the observation time range of WFCTA, established the LHSSAO-Model, and obtained the relevant fitting parameters. The difference between the MSIS-90 model and the measured data is mainly due to the fact that the model does not take into account some physical factors, such as the systematic deviation caused by the variation of atmospheric non-migratory tides and different bands of solar radiation.

Based on the above results, at LHAASO, the atmospheric depth values of the MSIS model from $4.4 \mathrm{~km}$ to $100 \mathrm{~km}$ are slightly larger than the measured values of the experimental data, but compared with the US standard atmosphere model, the agreement with 
the experimental data is higher,we will use Corsika simulations to analyze the differences in data performance between the model and American standard atmosphere model.

\section{Acknowledge}

This work is supported in China by the Fundamental Research Funds for the Central Universities (grant numbers 2682020CX77, 2682020CX73, 2682020CX74). It is also supported by the Science and Technology Department of Sichuan Province (grant numbers 2021YFSY0031, 2020YFSY0016), and by NSFC (grant number 11947404), and by National Key R\&D program of China (grant number 2018YFA0404201).

\section{References}

[1] He, Huihai. Design of the LHAASO detectors[J]. Radiation Detection Technology and Methods, 2018, 2(1):7.

[2] National Aeronautics and Space Administration. NASA Technical Memorandum. Washington D C, 1976. 74335

[3] NRLMSISE-00 empirical model of the atmosphere: Statistical comparisons and scientific issues. J Geophys Res, 2002, 107: 1468-1483

[4] WAN T,LIU $\mathrm{H}$ W,FAN J.Error band and confidence coefficient of atmospheric density models around altitude $100 \mathrm{~km}[\mathrm{~J}]$. Scientia Sinica(Physica,Mechanica Astronomica),2015,45(12):66-72.

[5] QIAO S,PAN W,BAN C,et al. Mesospheric density mea-sured by Rayleigh lidar over Golmud[J]. infrared Laser Eng.,2018,47(S1):S106005-1-6 Picone J M, Hedin A E, Drob D P, et al.

[6] Weijun Liu,Jiyao Xu,A. K. Smith,Wei Yuan. Comparison of rotational temperature derived from ground-based $\mathrm{OH}$ airglow observations with TIMED/SABER to evaluate the Einstein coefficients[J]. Journal of Geophysical Research: Space Physics,2015,120(11). 
Table 1: Fitting parameters of the atmospheric model

\begin{tabular}{|c|c|c|c|c|}
\hline Mouth & Altitude $\mathrm{h}(\mathrm{km})$ & $\mathrm{ai}\left(\mathrm{g} / \mathrm{cm}^{2}\right)$ & $\mathrm{bi}\left(\mathrm{g} / \mathrm{cm}^{2}\right)$ & $\mathrm{ci}(\mathrm{cm})$ \\
\hline \multirow{4}{*}{ January } & $4 \ldots 10$ & -70.19 & 1511.10 & 847861 \\
\hline & $10 \ldots 40$ & 0.7124 & 1427.82 & 622405 \\
\hline & $40 \ldots 70$ & -0.0198 & 477.32 & 791704 \\
\hline & $70 \ldots 100$ & -0.0006 & 234.98 & 653440 \\
\hline \multirow{4}{*}{ February } & $4 \ldots 10$ & -69.0042 & 1152.06 & 844628 \\
\hline & $10 \ldots 40$ & 0.7039 & 1425.44 & 622829 \\
\hline & $40 \ldots 70$ & -0.0232 & 453.49 & 800129 \\
\hline & $70 \ldots 100$ & -0.0007 & 2628.73 & 647197 \\
\hline \multirow{4}{*}{ March } & $4 \ldots 10$ & -68.4238 & 1147.93 & 846844 \\
\hline & $10 \ldots 40$ & 0.6739 & 1430.06 & 622480 \\
\hline & $40 \ldots 70$ & -0.0265 & 427.48 & 810334 \\
\hline & $70 \ldots 100$ & -0.0006 & 309.36 & 638828 \\
\hline \multirow{4}{*}{ April } & $4 \ldots 10$ & -68.8507 & 1140.99 & 854446 \\
\hline & $10 \ldots 40$ & 0.6719 & 1441.56 & 621523 \\
\hline & $40 \ldots 70$ & -0.0275 & 418.28 & 815759 \\
\hline & $70 \ldots 100$ & -0.0006 & 370.57 & 630105 \\
\hline \multirow{4}{*}{ May } & $4 \ldots 10$ & -70.3061 & 1137.10 & 865141 \\
\hline & $10 \ldots 40$ & 0.7112 & 1457.07 & 621232 \\
\hline & $40 \ldots 70$ & -0.0253 & 436.77 & 811871 \\
\hline & $70 \ldots 100$ & -0.0007 & 440.73 & 621805 \\
\hline \multirow{4}{*}{ June } & $4 \ldots 10$ & -72.1042 & 1137.32 & 875334 \\
\hline & $10 \ldots 40$ & 0.74637 & 1468.60 & 622606 \\
\hline & $40 \ldots 70$ & -0.02157 & 475.06 & 801687 \\
\hline & $70 \ldots \quad 100$ & -0.0007 & 497.69 & 615581 \\
\hline \multirow{4}{*}{ July } & $4 \ldots 10$ & -73.4723 & 1137.96 & 881852 \\
\hline & $10 \ldots 40$ & 0.7176 & 1152.28 & 625228 \\
\hline & $40 \ldots 70$ & -0.0199 & 509.74 & 792204 \\
\hline & $70 \ldots 100$ & -0.0007 & 510.33 & 613543 \\
\hline \multirow{4}{*}{ August } & $4 \ldots 10$ & -74.0548 & 1141.99 & 883373 \\
\hline & $10 \ldots 40$ & 0.6182 & 1467.63 & 627381 \\
\hline & $40 \ldots 70$ & -0.0186 & 519.68 & 788066 \\
\hline & $70 \ldots 100$ & -0.0006 & 463.06 & 617474 \\
\hline \multirow{4}{*}{ September } & $4 \ldots 10$ & -73.9856 & 1142.26 & 880356 \\
\hline & $10 \ldots 40$ & 0.5221 & 1460.86 & 627475 \\
\hline & $40 \ldots 70$ & -0.0192 & 507.51 & 788629 \\
\hline & $70 \ldots 100$ & -0.0006 & 3826.17 & 626778 \\
\hline \multirow{4}{*}{ October } & $4 \ldots 10$ & -73.5034 & 1141.61 & 873660 \\
\hline & $10 \ldots 40$ & 0.4991 & 1151.65 & 625561 \\
\hline & $40 \ldots 70$ & -0.0196 & 491.88 & 789852 \\
\hline & $70 \ldots \quad 100$ & -0.0006 & 2998.23 & 639809 \\
\hline \multirow{4}{*}{ November } & $4 \ldots 10$ & -72.7183 & 1142.66 & 864899 \\
\hline & $10 \ldots 40$ & 0.5630 & 1444.27 & 623294 \\
\hline & $40 \ldots 70$ & -0.0184 & 486.95 & 789097 \\
\hline & $70 \ldots 100$ & -0.0007 & 2472.46 & 650721 \\
\hline \multirow{4}{*}{ December } & $4 \ldots 10$ & -71.5869 & 1146.50 & 855582 \\
\hline & $10 \ldots 40$ & 0.6565 & 1435.27 & 622182 \\
\hline & $40 \ldots 70$ & -0.0181 & 487.27 & 788290 \\
\hline & $70 \ldots 100$ & -0.0007 & 2277.12 & 655417 \\
\hline
\end{tabular}

